\title{
La reforma del sistema de
}

\section{pensiones en Europa en el decenio de 1990: lecciones para América Latina}

\section{Louise Fox}

Economista Principal,

Banco Mundial

Lfox@Worldbank.org

\section{Edward Palmer}

Profesor de Economía

de Seguro Social,

Universidad de Uppsala

y Jefe de Investigación,

Junta Nacional de

Seguro Social,

Suecia

edward.palmer@abc.se
La reforma del sistema de pensiones europeo parte de diferen-

cias de ingresos relativamente bajas, debidas parcialmente a la alta cobertura del sistema. En América Latina persisten los problemas de informalidad e insuficiente cobertura, y la desigualdad de ingresos es elevada. Aun así, ambas regiones avanzan hacia esquemas de cuentas individuales con fuertes vínculos entre contribuciones y prestaciones. América Latina ha introducido cuentas financieras obligatorias. Europa se mueve hacia cuentas de reparto de por vida. Algunos países europeos han establecido sistemas de contribuciones definidas nocionales. En América Latina, mientras la cobertura sea insuficiente, estarán en primer plano la necesidad de garantías para los pobres y el problema específico de la pobreza y las mujeres. Otras cuestiones importantes son la edad mínima de jubilación y el riesgo moral asociado a esquemas que alientan o apoyan el retiro temprano. En relación con estos puntos y con los problemas de pobreza sería útil considerar cuidadosamente la experiencia europea. 


\section{Introducción}

Los sistemas públicos de pensiones se remontan en América Latina a la década de 1930. El diseño de los sistemas se calcaba del modelo entonces dominante: el europeo. En sus etapas iniciales, los sistemas latinoamericanos cubrían principalmente a los empleados del sector público y de las empresas transnacionales. En Europa en el decenio de 1930, con la posible excepción de los países mediterráneos, los sistemas tendían a tener una cobertura más amplia, aunque las prestaciones eran bajas. Como en Europa, los sistemas latinoamericanos se ampliaron en los decenios de 1950 y 1960. En general, sin embargo, siguieron siendo bastante elitistas, lo que se explica por el pequeño tamaño de la fuerza de trabajo del sector formal y la gran desigualdad de los ingresos. El papel preponderante del Estado en el proceso de industrialización de América Latina en la posguerra implicaba también que la fuerza laboral cubierta por el sistema se encontraba principalmente en el sector público. Como la fuerza trabajadora era joven y creciente, los sistemas podían solventarse.

Esta situación cambió a fines del decenio de 1970. La crisis del petróleo y la inflación consiguiente, el endeudamiento público para mantener a flote las economías y la crisis subsiguiente de la deuda en el decenio de 1980, seguida por una década de estabilización y lento crecimiento, quebrantaron la hacienda pública. La estabilización dejó al descubierto los problemas de sustentabilidad de los sistemas fiscales, cuando los compromisos de prestaciones no podían ya anularse con la inflación. También quedaron al desnudo las inequidades. En muchos países, un mismo historial de contribuciones resultaba en beneficios muy diferentes según la ocupación. Se introdujeron reformas en el sistema de pensiones para reducir las deudas del Fisco y mejorar la equidad del sistema. Las reformas latinoamericanas, que comenzaron en Chile y se difundieron a todo el continente, se plasmaron en un nuevo sistema público de pensiones: el de cuenta individual de contribución definida, con financiamiento totalmente anticipado y bajo administración privada (Banco Mundial, 1994).

Las reformas latinoamericanas influyeron en todo el mundo. A medida que en Europa crecía el convencimiento de que era necesario reformar el sistema, salían a colación frecuentemente los nuevos conceptos latinoamericanos. A la postre, Europa optó por seguir su propio camino en la reforma de los sistemas de pensiones, pero hay resabios del pensamiento latinoamericano en sus reformas del decenio de 1990. Y también allí el sistema de las cuentas individuales se considera hoy como la piedra angular de la reforma.

Aunque el modelo latinoamericano ha resistido bien el paso del tiempo, está comenzando a mostrar algunas grietas. Una de las principales es la de la cobertura (Holzmann, Packard y Cuesta, 2000). Se cifraban esperanzas generalizadas en que este aspecto mejoraría con la reforma, pero la experiencia de Chile muestra lo contrario. En consecuencia, no se espera que los sistemas latinoamericanos eviten la continuación de la pobreza en la vejez durante las próximas décadas. Frente a este desafío creciente, algunos miran hacia Europa para ver qué ha hecho el Viejo Mundo con la idea esencialmente neomundista de las cuentas individuales.

\section{II}

\section{La Organización de Cooperación y Desarrollo Económicos en el decenio de 1990}

Las pensiones públicas para la vejez se establecieron primitivamente en Europa, y en otras partes, para aliviar la pobreza durante esta etapa de la vida con una cobertura que abarcaba a toda la población trabajado- ra y a sus sobrevivientes. Aunque en general los países industrializados de alto ingreso tenían ya en el decenio de 1930 algún esquema de pensiones públicas, el nivel de vida de los ancianos seguía siendo motivo 
de gran preocupación política en el período de reconstrucción de la década de $1950 .{ }^{1}$ Muchos de los gobiernos tenían como prioridad establecer sistemas en beneficio de personas cuyo capital humano y ahorros habían sido destruidos por dos guerras y una crisis económica.

Durante los decenios de 1950 y 1960, en varios países aparecieron sistemas de pensiones públicos de prestaciones definidas, con reglamentos generosos. Algunos combinaban prestaciones comunes para todos con beneficios basados en el número de años de contribución; en algunos casos el número de años de cotización necesarios para lograr la prestación completa (por ejemplo, 30 años) era muy inferior a la duración normal de la vida de trabajo masculina en esa época, de 40-45 años, incluso a mediados del decenio de 1970 (Palmer, 1999). Además, las prestaciones mismas podían basarse en los mejores años $x$ de contribución del participante, o en sus últimos años de contribución $y$. No todos los años tenían la misma ponderación. Este tipo de fórmula de prestación elevó los beneficios para los trabajadores que se jubilaron en los decenios de 1960 y 1970, lo que era la meta de muchos países. A la larga, sin embargo, ha resultado un problema porque supone una redistribución injusta: resta más recursos a quienes han tenido una carrera más larga con ingresos relativamente parejos (típica de los empleos de servicio tanto en la categoría de operarios como de oficinistas) que a quienes han tenido una vida de trabajo más corta pero con grandes incrementos en sus remuneraciones (perfil típico de los profesionales y personal ejecutivo). Además, estos sistemas suelen ofrecer una pensión completa a una edad específica, y a veces también derechos por antigüedad que permiten a las personas con un número determinado de años de servicio jubilarse antes de llegar a la edad estipulada para percibir la pensión completa.

A medida que estas generosas disposiciones ${ }^{2}$ entraban en vigencia, descendía la participación masculina en la fuerza laboral en todos los países que originalmente formaban la Organización de Cooperación y Desarrollo Económicos (OCDE), salvo el Japón, y se reducía la edad de jubilación, en seis años en España, cinco en Francia, y cuatro años en Suecia, donde la edad para optar a la prestación completa se redujo en

\footnotetext{
${ }^{1}$ El informe Beveridge, publicado en 1942, fue encargado por Churchill con el propósito de mejorar la situación en el Reino Unido después de la guerra.

${ }^{2}$ Los sistemas de algunos países exigían sólo de 20 a 30 años de cobertura para obtener la totalidad de la prestación definida.
}

dos años en 1976. La esperanza de vida para ambos géneros a partir de los 60 años aumentó en alrededor de 2.5 años en el período 1975-2000. Así pues, ya sea que la prestación se pagara a un trabajador varón o a su cónyuge sobreviviente (por lo general uno o dos años menor y con una esperanza de vida cinco a seis años mayor), el período de la prestación se alargó en unos cinco a ocho años en el cuarto de siglo transcurrido desde mediados del decenio de 1970 (Palmer, 1999).

Hubo otro suceso en los decenios de 1960 y 1970 que tuvo repercusiones durante mucho tiempo. Alentados por el fuerte crecimiento económico de posguerra, se formó una alianza "solidaria" entre los sindicatos interesados en obtener buenas prestaciones para sus afiliados — desde temprana edad — y los empleadores que deseaban establecer un límite de edad contractual para los trabajadores más viejos a fin de eludir ciertos acuerdos sobre despido con los sindicatos ("último en entrar, primero en salir"). Esta alianza prohijó el concepto de un ciclo de vida con tres fases - educación, trabajo y jubilación- que apareció en el decenio de 1960-1970. La jubilación se transformó en una "fase de la vida" definida por las leyes y los contratos, acompañada con cierta frecuencia por beneficios contractuales que complementaban los sistemas estatutarios, con lo cual el egreso resultaba aún más atractivo.

Cuando existe al menos la opción teórica de optar entre el trabajo y la jubilación, en algunos países puede ser alto el gravamen implícito en prolongar la vida laboral comparado con el ingreso adicional que supone seguir trabajando. Evidencias de once países de la OCDE sugieren que los trabajadores actúan racionalmente y egresan de la fuerza laboral en edad más temprana si ven en ello alguna ventaja económica (Gruber y Wise, 1999). Se reconoce ahora en general que la edad real de jubilación tendrá que aumentar en los próximos decenios y que esto sólo podrá lograrse cambiando los sistemas de prestaciones para lograr la neutralidad actuarial con respecto al tiempo de la jubilación y la esperanza de vida en el momento de jubilar. Este hecho ha llevado a muchos países a inclinarse por las cuentas vitalicias, y a algunos (entre los europeos, Italia, Latvia, Polonia y Suecia) a introducir la esperanza de vida como un factor explícito del monto de las pensiones.

¿Y qué ha sucedido con la meta de aliviar la pobreza en la ancianidad? Mientras el ingreso se elevaba rápidamente, sobre todo durante los primeros 25 años del período de reconstrucción, fue posible transferir porcentajes crecientes del producto interno bruto 
(PIB) desde los trabajadores a los jubilados. No resultaba tan difícil financiar mejores prestaciones porque las personas pasaban la mayor parte de su vida adulta trabajando y menos tiempo jubilados. A medida que maduraban los sistemas, los ingresos de los jubilados eran cada vez mejores, en términos relativos.

Hacia mediados del decenio de 1990, el índice de pobreza entre los jubilados de la OCDE era bajo. Calculado como el 50\% del ingreso mediano disponible ajustado por el tamaño del hogar, poco menos de $14 \%$ de las personas de 65 años y más podían calificarse de pobres (Förster, 2000). En un extremo en que se encontraban Canadá, los Países Bajos y Suecia, la tasa de pobreza entre las personas de 65 años y más sólo llegaba al 3\%, empleando la medida del $50 \%$ del ingreso mediano. Es interesante señalar que hay países que logran resultados muy parecidos con combinaciones muy distintas de sistemas privados y públicos (OCDE, 2000).

Los países de la OCDE exhiben diferentes resultados en cuanto a pobreza. Por ejemplo, en los Estados Unidos, con una red de seguridad menos generosa, una inmigración considerable y una mayor dispersión en la distribución del ingreso, alrededor de $20 \%$ de las personas de 65 años y más podían calificarse como pobres en 1993 (Förster, 2000). Sin embargo, la pobreza en los Estados Unidos, definida como el 50\% del ingreso mediano, permite un nivel de vida alto si se le compara con el de los países más pobres dentro y fuera de la OCDE. Hecha esta advertencia, observamos que la tasa de pobreza se situaba en torno al $20 \%$ en Turquía y el $30 \%$ en Grecia.

En resumen, los sistemas de la OCDE que se diseñaron primitivamente para dar mejores prestaciones a las personas que se jubilaban en los primeros decenios de la posguerra pudieron financiarse hasta el decenio de 1990 gracias a las normas de transición, las modalidades de trabajo y una menor esperanza de vida. El modelo vinculado al ciclo de vida - con sus fases de educación, trabajo y jubilación- se caracteriza por edades de retiro más tempranas que, al combinarse con una mayor longevidad, prolongaron la fase de jubilación. Como resultado, los costos de las pensiones subieron dramáticamente, con la perspectiva de que ocurrieran cambios aún más significativos a medida que continuara el envejecimiento de la población. En conclusión, los países pudieron transferir la creciente prosperidad a los ancianos, pero al mismo tiempo el diseño del sistema y las normas tributarias actuaron en contra de la posibilidad de sustentación financiera. A fines del decenio de 1980 muchos países de la OCDE se dieron cuenta de que debían reestructurar sus sistemas de reparto (pay-as-you-go systems).

\section{III}

\section{Países latinoamericanos y países de alto ingreso en el decenio de 1990}

En el medio siglo posterior a la segunda guerra mundial, los países de alto ingreso de la $\mathrm{OCDE}^{3}$ evolucionaron desde economías industriales a economías posindustriales, caracterizadas por un alto grado de urbanización, con economías de mercado formales, apoyo decreciente en las estructuras de la familia ampliada y participación cada vez mayor de las mujeres en el mercado del trabajo. Estas tendencias económicas y culturales se cimentaban en instituciones jurídicas que funcionaban bien y en la administración fiscal eficiente de los impuestos, las contribuciones y las

\footnotetext{
${ }^{3}$ Alemania, Australia, Austria, Bélgica, Canadá, Dinamarca, España, Estados Unidos, Finlandia, Francia, Irlanda, Italia, Japón, Nueva Zelandia, Noruega, los Países Bajos, el Reino Unido, Suecia y Suiza.
}

transferencias. Hay algunas similitudes pero también algunas diferencias importantes entre los países de alto ingreso de la OCDE y los países de América Latina en estos aspectos. Consideraremos aquí algunas de ellas.

En general, la universalidad es la característica clave de los sistemas de pensiones en casi todos los países de la OCDE, aunque debe reconocerse que dicho concepto presenta connotaciones distintas en cada uno de ellos. Algunos tienen regímenes especiales para diversas ocupaciones, incluso el trabajo por cuenta propia. Como ejemplo podemos citar a Italia que, además del trato especial para los trabajadores por cuenta propia, mantiene para algunas ocupaciones regímenes de reparto que dejaron de autofinanciarse. En el decenio de 1990, este país se fue acercando a la verdadera universalidad, cubriendo incluso a los trabajadores por 
cuenta propia. Otros países no tienen excepciones (por ejemplo, los escandinavos, el Reino Unido y los Estados Unidos). Australia e Irlanda, en cambio, utilizan sistemas basados en el empleador. En Australia éstos se volvieron obligatorios en 1992 pero todavía con el respaldo de una red de seguridad pública.

El cumplimiento de las normas legales en los países de alto ingreso de la OCDE se ha visto facilitado por el aumento de las empresas grandes y medianas y de los empleadores gubernamentales y por la disminución del empleo por cuenta propia. A medida que el mercado del trabajo se orienta hacia esquemas contractuales más flexibles y en estos últimos tiempos se aleja de los contratos, es posible que en el futuro la OCDE también se enfrente a un entorno laboral que facilite la evasión. La legitimidad del sistema público de pensiones depende de que la opinión pública crea que el contribuyente está pagando la parte que le corresponde. La lógica tras la economía informal (que no cumple con las disposiciones legales) es que algunos pagan, generalmente por intermedio de sus patrones, mientras que otros - los que se hallan en la economía informal - no pagan, y a la larga los que pagan financian a los que no pagan. Los sistemas que establecen un fuerte vínculo entre las prestaciones y las contribuciones son atractivos porque respaldan la legitimidad de aquellos que pagan.

Los sistemas de pensiones en muchos de los países de América Latina son en principio de cobertura universal, como en Europa y América del Norte, pero la baja tasa de cumplimiento de la normativa y la existencia de la economía informal implican que en la práctica su cobertura es baja. Según el Banco Interamericano de Desarrollo (BID, 1998), en América Latina el sector informal representa en promedio un $27 \%$ de la economía, pero presenta grandes variaciones entre países que van del $10 \%$ al $60 \%$. Una mayor formalización puede aumentar o disminuir la desigualdad, según cuál haya sido el punto de partida: hasta cierto umbral puede agudizarla, pero de allí en adelante el mejoramiento continuado ayudará a reducirla. El BID ha calculado que ese umbral se sitúa a la altura del $25 \%$ a $30 \%$ de informalidad. Según el estudio citado, más o menos la mitad de los países latinoamericanos se encuentran ahora en el punto en que una mayor formalización presumiblemente reduciría la desigualdad. ${ }^{4}$ En otras palabras, los impuestos, las contribucio-

\footnotetext{
${ }^{4}$ Nótese que la desigualdad medida por el coeficiente de Gini suele ser inferior a 0.3 en los países de altos ingresos, donde los mercados son formales, pero suele ser de 0.5 o más en los países latinoamericanos.
}

nes y las transferencias o son neutrales o son progresivos, y su redistribución va más bien en beneficio de las clases más pobres más que de las más ricas.

Los sistemas de cuentas financieras que se popularizaron en América Latina en el decenio de 1990, así como también los sistemas de contribuciones definidas nocionales $(\mathrm{NDC})^{5}$ que aparecieron en Europa durante el mismo período, redistribuyen los recursos individuales del participante a lo largo de su vida. En principio, en ellos se elimina el elemento de gravamen que contienen los sistemas tradicionales de prestaciones definidas, con lo cual las prestaciones se vinculan directamente con las contribuciones. Como corolario, la política de redistribución se traslada al sistema general de transferencias de impuestos.

Los sistemas de cuentas financieras introducidos por muchos países latinoamericanos en el decenio de 1990 son un medio para transferir recursos individuales a lo largo del ciclo de vida, pero también permiten desarrollar mercados financieros (Holzmann, 1997). La enseñanza que todavía le puede transmitir Europa a América Latina es que los regímenes de reparto también pueden emular a los sistemas de cuentas financieras cuando se conciben como sistemas de contribuciones definidas nocionales.

La estructura de la producción y el grado de urbanización en América Latina se parecen asombrosamente a los de los países de alto ingreso de la OCDE (cuadro 1), ${ }^{6}$ pero sólo desde este punto de vista; los aspectos en que difieren ambos grupos de países se relacionan principalmente con el financiamiento de las pensiones. Como el crecimiento de la fuerza de trabajo es mucho mayor en América Latina y la fecundidad continúa siendo bastante más alta que dos hijos por mujer, esta región seguirá generando una fuerza de trabajo creciente durante algún tiempo más. Además, sigue habiendo mucho campo para el incremento de la participación femenina en la fuerza laboral. Por otro lado, como lo muestra la experiencia de la OCDE, a medida que las mujeres se dediquen más a su propia carrera y tengan sus propios emolumentos, tendrán menos hijos. En resumen, las estadísticas demográficas dicen que América Latina se encuentra en mucho

\footnotetext{
${ }^{5} \mathrm{NDC}=$ notional defined contribution.

${ }^{6}$ En este texto, los países de alto ingreso son: Alemania, Australia, Austria, Bélgica, Canadá, Dinamarca, Emiratos Árabes Unidos, España, Estados Unidos, Finlandia, Francia, Hong Kong, Italia, Irlanda, Israel, Japón, Kuwait, Noruega, los Países Bajos, Nueva Zelandia, el Reino Unido, Singapur, Suecia y Suiza. Los datos son algo antiguos (de 1970) y es probable que las regiones se hayan acercado desde entonces.
} 
CUADRO 1

Países de alto ingreso de la OCDE y países de América Latina y el Caribe: Algunos datos de 1970

(Porcentajes)

\begin{tabular}{|c|c|c|c|c|}
\hline Indicadores & \multicolumn{2}{|c|}{ Países de alto ingreso } & \multicolumn{2}{|c|}{$\begin{array}{c}\text { Países de América Latina } \\
\text { y el Caribe }\end{array}$} \\
\hline 1. Estructura de producción & 1970 & & 1970 & \\
\hline Agricultura & 12 & & 4 & \\
\hline Industria & 35 & & 38 & \\
\hline Servicios & 53 & & 58 & \\
\hline 2. Fuerza trabajadora & $1980-1993$ & $1993-2000$ & $1980-1993$ & $1993-2000$ \\
\hline Crecimiento medio anual $(\%)$ & 0.7 & 0.3 & 2.5 & 2.3 \\
\hline 3. Empleo & 1970 & 1993 & 1970 & 1993 \\
\hline Participación femenina en fuerza trabajadora & 36 & 38 & 22 & 27 \\
\hline 4. Urbanización & 1970 & 1993 & 1970 & 1993 \\
\hline En \% de la población & 74 & 78 & 57 & 71 \\
\hline 5. Tasa de fecundidad & 1970 & 1993 & 1970 & 1993 \\
\hline & 2.3 & 1.7 & 5.2 & 3.1 \\
\hline
\end{tabular}

Fuente: Banco Mundial (1995).

mejor situación que la OCDE para afrontar los primeros 25 años del siglo XXI, pero que con el descenso de la fecundidad, incluso América Latina tendrá que enfrentar inevitablemente la situación que ahora tienen ante sí los países de la OCDE, aunque en una época mucho más tardía.

Otra característica que distingue a los países latinoamericanos de los de la OCDE es la prevalencia relativa de hogares ampliados. En los Estados Unidos, por ejemplo, sólo alrededor del $15 \%$ vive en hogares de este tipo mientras que un $3 \%$ vive en hogares unipersonales. En América Latina el cuadro es muy distinto. De un $40 \%$ (Brasil) a un 55\% (Argentina, Perú y Venezuela) de la población vive en hogares ampliados y sólo cerca del 5\% en hogares unipersonales (BID, 1998). Esto sugiere que las transferencias dentro de la familia ampliada tienen importancia en América Latina y que desempeñan un papel significativo en aliviar la pobreza, sobre todo en la ancianidad. Con la continuación del crecimiento económico y la mayor formalización, podría suceder que la situación en América Latina llegara a parecerse más a la que predomina en los países de alto ingreso de la OCDE, aunque se plantea también la pregunta - ya de índole cultural y con variadas respuestas, incluso dentro de la OCDE- si las personas preferirán vivir con la familia ampliada aunque tengan los medios para vivir solas.

En suma, América Latina reúne las condiciones previas para elevar su productividad en los próximos veinticinco años merced al crecimiento de su capital humano y de su fuerza de trabajo. El desafío está en involucrar a toda la población en este proceso de crecimiento e incluirla en las instituciones de la economía formal y en sistemas de pensiones que transfieran los recursos individuales a lo largo del ciclo de vida del individuo. El proceso de formalización implica incluir a la mayoría de la población en esos sistemas de pensiones. En los países de alto ingreso de la OCDE el crecimiento económico ha sido condición previa para la reducción efectiva de la pobreza en la ancianidad, pero ha tenido suma importancia también la existencia de mercados del trabajo muy formalizados, con un alto grado de cumplimiento de las leyes tributarias y otras condiciones legales. 


\section{IV}

\section{Anuncios de tormenta en la OCDE: lecciones para América Latina}

El centro de interés en los debates sobre los sistemas públicos de pensiones en la OCDE se desplazó paulatinamente de la suficiencia del sistema a su capacidad de sustentación financiera y la equidad entre generaciones. Hacia 1990, en gran parte de la OCDE la reforma del sistema de pensiones se discutía en torno a estos dos últimos aspectos. El debate y las medidas adoptadas al respecto por los países podrán ser útiles para América Latina.

La lógica de los sistemas de reparto es sencilla: el incremento de los gastos debe estar respaldado por un financiamiento mayor a fin de mantener el equilibrio financiero. En un medio estable, sin cambios de fecundidad, mortalidad específica por edades o inmigración neta, serían estáticos el tamaño y la composición de la población. Si además se mantuvieran constantes las modalidades de trabajo y la estructura etaria de la fuerza laboral, los sistemas de reparto no tendrían problemas financieros serios a largo plazo. Pero el futuro financiero de estos sistemas en la OCDE se empezó a ver dudoso porque todos esos parámetros estaban cambiando y se preveía que seguirían cambiando en el sentido de representar una mayor carga financiera para los sistemas de pensiones. La fecundidad ha caído muy por debajo del nivel requerido para reproducir a la población, los índices de mortalidad están bajando y ha estado disminuyendo la participación en la fuerza laboral de los trabajadores de más edad, sobre todo entre los varones.

Por efecto de las bajas tasas de fecundidad, el crecimiento de la fuerza de trabajo se volverá negativo en el decenio de 2000 y se mantendrá así en la mayoría de los países de alto ingreso de la OCDE. En realidad, aunque en el decenio indicado aumentara la fecundidad por encima de los dos hijos por mujer necesarios para reproducir a la población, este mayor contingente sólo llegaría a la edad productiva en 20252035. Se calcula que Europa tendrá que fomentar la inmigración de unos 30 millones de personas en los próximos treinta años para conservar una fuerza de trabajo parecida a la que tiene. A largo plazo, sin embargo, el problema no se resolverá ni siquiera con un incremento de una vez por todas de esa fuerza.
Mientras se mantenga la fecundidad global por debajo de la necesaria para reproducir la población, será necesaria una inmigración neta permanente para mantener una fuerza laboral de un tamaño determinado.

Aunque el PIB real aumentó alrededor de 3\% anual desde 1970 hasta mediados de los años noventa (Banco Mundial, 1995), el crecimiento de la base contributiva no bastó para evitar que las cotizaciones de los sistemas de reparto tuvieran que elevarse para cubrir los déficit de liquidez. Al llegar al decenio de 1990, las tasas elevadas y crecientes de contribución preocupaban no sólo por el costo futuro de las pensiones y la sustentabilidad a largo plazo de obligaciones basadas en las normas vigentes, sino también por sus posibles efectos de largo plazo sobre el empleo y el cumplimiento de la normativa legal. Lo primero por el efecto de la proporción de impuestos incorporada en los sistemas de prestaciones definidas tradicionales, y lo segundo, por la inclinación natural de los individuos y los empleadores a buscar resquicios para evitar el pago de contribuciones cuando las tasas son altas.

Las mayores presiones sobre los sistemas de pensiones para la vejez de reparto en la OCDE provienen de dos factores: la longevidad tiende a aumentar y los trabajadores más viejos tienden a abandonar el trabajo a edades más tempranas, frecuentemente recurriendo al sistema público de compensación por incapacidad. En la OCDE, las proyecciones indican que los mayores de 60 años aumentarán desde alrededor de $20 \%$ de la población al terminar el decenio de 1990 , a $27 \%$ en 2020 y $30 \%$ en 2030 (cuadro 2). La longevidad ha aumentado a ritmos diferentes en los distintos países de la OCDE y con rapidez mayor entre las mujeres que entre los hombres. Sin embargo, en épocas recientes ha habido incrementos rápidos de la longevidad masculina gracias a los grandes avances en el tratamiento de las enfermedades cardiovasculares, que han permitido ganar años de vida de buena calidad.

Por ejemplo, en Alemania, los Estados Unidos y Suecia el incremento medio de la esperanza de vida para hombres y mujeres a los sesenta años representa un año de vida más por cada diez años transcurridos a partir de 1960. En Alemania, esto significa que a los 
CUADRO 2

Organización de Cooperación y Desarrollo Económicos (OCDE): Proporción de la población que tiene más de 60 años (Porcentajes)

\begin{tabular}{lrrrrr}
\hline & 2000 & 2010 & 2020 & 2030 & 2050 \\
\hline OCDE & 19.9 & 23.1 & 27.0 & 30.7 & 31.2 \\
& & & & & \\
Países en transición & 17.0 & 18.2 & 21.5 & 22.7 & 26.6 \\
Países latinoamericanos & 7.7 & 9.3 & 12.2 & 16.0 & 23.5 \\
Asia & 7.3 & 8.6 & 11.6 & 15.0 & 20.7 \\
Oriente medio y & & & & & \\
África del norte & 7.3 & 8.1 & 10.0 & 12.4 & 18.1 \\
África al sur del Sahara & 4.4 & 4.5 & 4.9 & 5.9 & 9.9 \\
\hline
\end{tabular}

Fuente: Banco Mundial (1998).

60 años la esperanza de vida aumentó de 17 a 21 años, en Suecia de 18 a 22 años, en los Estados Unidos de unos 17.5 a 21.5 años, y en Japón, de poco más de 16 años a 22. Los demógrafos esperan que la longevidad siga elevándose a un ritmo más o menos similar, aunque este pronóstico puede quedarse corto, ya que se basa más en la evolución histórica que en el análisis del impacto de nuevas tecnologías médicas y cambios en los hábitos de vida.

La fórmula siguiente es útil para ilustrar el predicamento en que se halla el sistema de reparto:

$$
\text { Tasa de contribuciones }=\frac{\bar{p}}{\bar{w}} * \frac{\text { jubilados }}{\text { contribuyentes }}
$$

Si se desea mantener un coeficiente predeterminado entre la prestación media $p$ y el salario medio $w$ a fin de que la contribución permanezca constante, el número de contribuyentes debe aumentar a la misma tasa que el número de jubilados. Si la longevidad aumentara en un año por cada diez que transcurran en el futuro, se producirá un aumento de 20\%-25\% en los próximos 40 años. Con un número constante de contribuyentes, habría que elevar las cotizaciones en el mismo porcentaje. El problema es que el crecimiento de la fuerza de trabajo autóctona será negativo, y si continúa la tendencia a recurrir a la jubilación anticipada por incapacidad, la situación será aún más grave. En suma, la OCDE no puede permitir que disminuya la fuerza laboral si quiere mantener sus jubilaciones medias como están con respecto al salario medio - es decir, si las contribuciones siguen casi al mismo nivel de ahora.

También hay una presión considerable para que se reduzca la edad de retiro. En muchos países, los trabajadores y los empleadores han abusado de normas de retiro generosas y de sistemas de compensación aparentemente sin límites ante una posible incapacidad de trabajadores mayores. Como ya hemos señalado, la edad media de egreso de la fuerza laboral ha descendido en los últimos treinta años. Aunque la edad para recibir la pensión completa por vejez en muchos países de la OCDE suele ser la de 65 años, la edad media en que los individuos dejaban de trabajar a mediados del decenio de 1990 no superaba los 56-57 años entre las mujeres y los 59 entre los hombres.

Hay muchos indicios de que la tendencia al egreso prematuro de la fuerza de trabajo no se justifica por razones de salud en los países de alto ingreso de la OCDE. En general, el entorno del trabajo industrial mejoró enormemente en la última mitad del siglo XX y, por añadidura, sólo alrededor de un tercio de la fuerza laboral se dedica a la industria. Las pensiones por incapacidad física han aumentado dramáticamente en los últimos treinta años, ${ }^{7}$ mientras que ha permanecido constante o ha disminuido el número de personas en empleos que exigen un gran desgaste físico. ${ }^{8}$

En parte, la jubilación temprana de los trabajadores de más edad obedece a incentivos económicos. ${ }^{9}$ Otro elemento que fomenta los retiros por incapacidad entre los trabajadores mayores surge cuando los empleadores necesitan reducir personal. Sobre todo en Europa, parece haber un consenso generalizado entre las partes del contrato social (administración, sindicatos y gobierno) de que ésta es una salida legítima para los trabajadores más viejos. Como resultado de este consenso implícito, los empleadores, con el apoyo de los sindicatos, se desinteresan del mejoramiento del entorno laboral y la capacitación en el trabajo de los empleados más antiguos. Y ello pese a que en promedio las exigencias especiales para los trabajadores de mayor edad no son muchas y cuesta poco proporcionarlas (Ilmarinen, 1999). El beneficio para los empleadores de deshacerse de los trabajadores más viejos es una pérdida de bienestar social para la nación. Disminuyen las personas ocupadas y los que tienen empleo deben pagar más impuesto por la salida prematura de otros. El beneficio para los empleadores también revela poca visión. Cuando escasea la mano de obra, este

\footnotetext{
${ }^{7}$ Véase, por ejemplo, Aarts, Burkhauser y de Jong (1996).

${ }^{8}$ Por ejemplo, Steuerle, Spiro y Johnson (1999) describen el caso de Estados Unidos, y NSIB (2001) el de Suecia.

${ }^{9}$ Este es el tema tratado en Gruber y Wise (1999).
} 
comportamiento agota la oferta y aumenta el costo del trabajo.

En resumen, el aumento de la longevidad, la declinación de la fecundidad y la práctica actual de desalojar a los trabajadores más viejos de sus puestos de trabajo se confabulan contra el financiamiento de las pensiones. Esta última tendencia puede frustrar el ver- dadero propósito de las pensiones por incapacidad, cual es el de proporcionar un ingreso satisfactorio para personas que han sufrido enfermedades o accidentes muy serios. El cambio demográfico adverso y la presión de los costos resultante del uso abusivo de sistemas generosos impulsaron las reformas que implantó la OCDE en el decenio de 1990.

\section{$\mathrm{V}$}

\section{El surgimiento de sistemas de reparto con contribuciones definidas nocionales}

El objetivo de las reformas efectuadas por la OCDE en el decenio de 1990 era crear un sistema de mayor equidad entre generaciones. Esto se logró desplazando una mayor parte del riesgo a los trabajadores mientras están activos, y avanzando hacia el uso de las remuneraciones de toda su vida laboral como base para calcular las prestaciones. Esto se traduce en menores prestaciones por menos trabajo, pero, por lo general, también en ganancias actuariales por permanecer en la fuerza de trabajo.

Un segundo objetivo era crear un sistema de mayor equidad intrageneracional. Los sistemas que se basan en los ingresos de toda una vida laboral también cumplen con esta condición. El sistema de reparto con contribuciones definidas nocionales adoptado en Italia (1995) y Suecia (1994) lleva este concepto a su plenitud en un modelo de contribuciones definidas basadas en los ingresos de toda la carrera laboral, que toma en cuenta también las variaciones en la esperanza de vida al calcular el monto de las pensiones.

¿Qué es el sistema de reparto con contribuciones definidas nocionales? Es un sistema en el cual los asalariados pagan contribuciones según una tasa fija, las que se acreditan a sus cuentas individuales nocionales- éste es el aspecto de contribución definida del sistema. Las personas pagan contribuciones sobre sus emolumentos durante todo el tiempo que trabajen. Los valores contables del año anterior se recalculan anual- mente aplicándoles un índice nominal del salario por empleado en Suecia ${ }^{10}$ y del PIB en Italia. La información sobre cambios en la esperanza de vida y, sobre la base del registro contable individual, su efecto sobre una pensión reclamada a determinada edad, se encuentra disponible en cualquier momento en Suecia. En otras palabras, los cambios en la esperanza de vida son un parámetro que los individuos pueden tomar en cuenta en el momento en que deciden cuándo y cómo dejar de trabajar. En principio, el sistema de contribuciones definidas nocionales permite solicitar cualquier porcentaje de una prestación en cualquier momento después de cumplida la edad legal mínima para optar a ella. Las personas pueden combinar una prestación parcial o completa con el trabajo (y continuar contribuyendo y adquirir nuevos derechos) y pueden así obtener una prestación recalculada en alguna fecha posterior.

Por último, cabe señalar que los aspectos redistributivos no han sido olvidados. Todos los países han establecido alguna especie de pensión de garantía o de asistencia social, previa comprobación de la insuficiencia de medios de quien la solicita, para proveer de algún ingreso a los que llegan a la vejez en la pobreza.

\footnotetext{
${ }^{10}$ Esto se suplementa, sin embargo, con un piso que se basa en la masa del salario de contribución, el cual afectará tanto la indización del capital nocional como el pago de beneficios.
} 


\section{VI}

\section{Sistemas de cuentas financieras}

A fines del decenio de 1980, el Reino Unido fue el único de los países de la OCDE que trasladó parte del sistema de seguro obligatorio de reparto al sistema de cuentas financieras. En 1992 Australia hizo obligatorios los sistemas privados de jubilación basados en las iniciativas de los empleadores y en 1994 Suecia aprobó una ley que hacía obligatorio el sistema de cuentas financieras en adición a su nuevo sistema de contribuciones definidas nocionales. El Reino Unido y Suecia mantuvieron los principios de reparto en sus sistemas. ${ }^{11}$ Australia mantuvo su programa general de apoyo para la vejez financiado con el erario nacional. ${ }^{12}$

Otros países que establecieron sistemas de cuentas financieras en el decenio de 1990 como parte del sistema de previsión obligatorio han retenido grandes segmentos (pilares) de reparto (por ejemplo Argentina, Hungría y Polonia). Europa no ha seguido el ejemplo de Chile de eliminar el elemento de reparto, aunque tanto Hungría como Polonia han adoptado el modelo administrativo chileno para el diseño del sistema de cuentas financieras. Suecia tampoco ha seguido el ejemplo de Chile y los países que han legislado más recientemente sobre el sistema de cuentas financieras han tendido a seguir el diseño de Suecia (por ejemplo Croacia y Latvia). En esta sección explicaremos por qué Europa ha tomado este camino.

\section{Financiamiento de la transición a los sistemas de cuentas financieras}

¿Por qué no son más numerosos los países que han seguido el ejemplo de Chile y han eliminado por com-

\footnotetext{
${ }^{11}$ Suecia mantuvo un componente de reparto importante - las contribuciones definidas nocionales representaban $16 \%$ y las cuentas financieras individuales $2.5 \%$ de la tasa total de contribución-, pero para gran parte de la fuerza de trabajo esto se complementó con un nuevo sistema de cuentas financieras basado en las ocupaciones que implicó nuevas tasas de cotización de 2\%-4\% (por ejemplo, $3 \%$ para los operarios). Así, un operario joven tiene ahora un $65 \%$ de la tasa de contribución total en cuentas financieras individuales.

12 Desde 1992 se exige a los empleadores en Australia establecer una cuenta de jubilación y contribuir a ella un porcentaje mínimo (que subió de 3\% en 1992 a 9\% en 2002) para sus empleados. Las personas pueden complementar estas sumas voluntariamente. El sistema cubre alrededor de $35 \%$ de los trabajadores por cuenta propia y $95 \%$ de los empleados con jornada completa. En 1995 abarcaba a alrededor de $80 \%$ de todos los ocupados.
}

pleto el sistema de reparto? Se señalan por lo menos dos razones. En primer lugar, en los años anteriores a la reforma, Chile había recortado el gasto público creando un superávit de 5.5\% del PIB que se aprovechó para cubrir la mayor parte de la deuda derivada de la transición. Sólo alrededor de $1.5 \%$ del PIB por año representaba una carga doble. ${ }^{13}$ Por otro lado, ni Australia ni Kazakstán tenían grandes compromisos con el sistema de reparto que sería reemplazado y, por lo tanto, en un comienzo no había un problema de conversión. En el caso de Australia, la reforma de 1992 simplemente ordenó la instauración de sistemas patronales que ya estaban bastante difundidos. La obligación suponía que todos los empleadores debían apartar fondos para sus empleados y que la tasa de contribución tenía que ceñirse como mínimo a la estipulada.

Los países de la OCDE y los de transición, pero también algunos latinoamericanos, presentan un perfil demográfico de mayor edad que Chile en la época de la reforma. En la OCDE los derechos adquiridos tienen un mayor peso. Los derechos adquiridos que había que monetizar con una reforma grande y completa hacia el sistema de cuentas financieras habrían sido cuantiosos, así como los impuestos requeridos para hacerlo.

Hay también una segunda razón, más de fondo, que explica por qué países que introdujeron la reforma después de Chile han optado por no abandonar por completo su sistema de reparto. Tanto el sistema de reparto como el de cuentas financieras se asocian con riesgos de fase descendente y al combinar estos dos pilares en la cartera de la previsión social se ofrece una mayor seguridad. En Europa, tanto Suecia como Polonia ${ }^{14}$ esgrimieron argumentos de este tipo.

¿De qué forma han podido los países encontrar los medios para establecer los sistemas con financiamiento anticipado? Se han usado diversos mecanismos para introducir los sistemas de cuentas financieras como segundo pilar. Entre ellos figuran la reducción de los compromisos con los jubilados bajo el sistema de

\footnotetext{
${ }^{13}$ Valdés-Prieto, 1997, p. 205. Obsérvese que esto representa 3.75\% de la nómina salarial asegurada, suponiendo que los salarios cubiertos representan $40 \%$ del PIB.

${ }^{14}$ El lema de la reforma polaca era "La seguridad mediante la diversidad".
} 
reparto, por lo general cambiando las fórmulas de indización; la reducción de los compromisos con los trabajadores activos, introduciendo normas más estrictas para otorgar las prestaciones; aprovechamiento de los ciclos demográficos; traslado de otros activos al sistema de jubilaciones para ayudar a financiar la transición; cobro de contribuciones mayores que las que se acreditaban a las cuentas individuales (es decir, imposición de gravámenes), y reducción de otros gastos de consumo gubernamentales, creando así un superávit presupuestario para ayudar a financiar los compromisos anteriores del sistema de reparto (Fox y Palmer, 2001).

Muchas de las reformas para introducir el sistema de cuentas financieras han aprovechado uno o más de estos arbitrios al mismo tiempo. Una combinación típica consiste en reducir los compromisos con los trabajadores activos, aprovechar los ciclos demográficos y financiar parcialmente con impuestos. Lo primero se ha logrado adoptando el concepto de remuneraciones de toda la vida laboral en la fórmula de reparto con beneficios definidos, elevando la edad para optar a una pensión mínima o completa $\mathrm{y}$, en el caso de los países que introducían sistemas de contribuciones definidas nocionales, tomando en cuenta la esperanza de vida - a la fecha de jubilación - al calcular la pensión vitalicia. Como ejemplo cabe citar Hungría, Latvia, Polonia y Suecia, pero sólo los tres últimos han aplicado sistemas de contribuciones definidas nocionales. Los países aprovechan las ventajas demográficas al reformar a buen tiempo antes de que una gran cohorte llegue a la edad de jubilar. Esta es una ventaja típica en Europa oriental, donde la explosión de nacimientos ocurrió como diez años más tarde que en el resto de la ocDE. Por último, algunos países europeos, igual que Chile, han aceptado que la introducción de un sistema de cuentas financieras obligatorio puede necesitar el apoyo financiero del Fisco, por lo menos en cierto grado.

Bolivia y Polonia son ejemplos de países que han transferido otros activos al sistema. En Suecia las grandes reservas del sistema de reparto ayudarán a pagar por la explosión demográfica de los años cuarenta, con lo cual los costos de transición se mantendrán dentro de los límites de una tasa fija de contribuciones definidas nocionales junto con el pilar de apoyo financiero. La introducción de las contribuciones definidas nocionales disminuyó las obligaciones a futuro de Suecia en el momento requerido para introducir gradualmente el sistema de cuentas financieras, reduciendo las prestaciones para las cohortes juveniles en el sistema de reparto pero dándoles una mayor participación en el nuevo sistema de cuentas financieras.
En Chile, Australia y Kazakstán, los sistemas de cuentas financieras se combinan con varias garantías. En Australia hay una pensión mínima universal previa comprobación de insuficiencia de ingresos y activos. Chile tiene una pensión mínima garantizada para los trabajadores asegurados, así como una de asistencia social para los indigentes que no están asegurados. Kazakstán también ha establecido una pensión mínima garantizada para los trabajadores asegurados.

Por último, cabe señalar que la reforma conlleva algunos costos que los países pueden haber subestimado. En los países en que la cobertura es escasa, el costo futuro de la garantía podría ser muy alto, lo que implicaría un alza en la tasa impositiva para los trabajadores futuros. Aun en países con garantías, los costos pueden estar subestimados. Por ejemplo, la pensión mínima garantizada en el Reino Unido y Suecia está amarrada a un índice de precios. En el Reino Unido ya se presiona por elevar el mínimo. Por otro lado, el argumento para separar el elemento de redistribución del elemento de seguridad social se basa en la hipótesis de que las propuestas de modificar las garantías pueden sopesarse con la opción de recurrir a los impuestos dentro del proceso político normal.

\section{El establecimiento de los sistemas de cuentas financieras}

Chile (1981), el Reino Unido (1986), (Australia (1992) y Suecia (1995) representan cuatro modelos de cómo establecer sistemas de cuentas financieras que han evolucionado en los últimos veinte años (cuadro 3 ). Los países que instauraron el sistema de financiamiento anticipado en los años noventa tienden a seguir uno u otro de estos modelos. Hay varios problemas que se plantean al diseñar modelos de cuentas financieras. Algunos de ellos se describen y analizan en Palmer (2000).

Se suele preguntar si los sistemas de seguridad social debieran permitir a los beneficiarios la libre elección de fondos o si debieran restringir sus opciones, por ejemplo a un número pequeño de fondos reajustables. Este último es un punto de vista que sostienen muchos economistas en los Estados Unidos, quienes creen que el sistema de seguridad social para la vejez en ese país debiera reorganizarse total o parcialmente con miras a establecer un financiamiento anticipado. Este punto de vista se apoya en el hecho de que así se reduce a un mínimo el riesgo cuando se permite a los individuos que elijan sus propios fondos, y que a la vez se obtiene una rentabilidad indizada por 


\begin{tabular}{|c|c|c|c|c|c|c|c|}
\hline & $\begin{array}{l}\text { ¿Quién elige al } \\
\text { administrador } \\
\text { del fondo de } \\
\text { pensiones? }\end{array}$ & $\begin{array}{l}\text { ¿Quién cobra las } \\
\text { contribuciones? }\end{array}$ & $\begin{array}{l}\text { ¿Quién lleva la } \\
\text { contabilidad/ } \\
\text { quién envía los } \\
\text { informes } \\
\text { financieros? }\end{array}$ & $\begin{array}{l}\text { Número de } \\
\text { fondos/ ¿quién } \\
\text { elige los } \\
\text { fondos? }\end{array}$ & $\begin{array}{l}\text { ¿Quién elige al } \\
\text { proveedor de la } \\
\text { pensión? }\end{array}$ & $\begin{array}{l}\text { Administrador } \\
\text { de los seguros }\end{array}$ & $\begin{array}{l}\text { Formas del } \\
\text { beneficio }\end{array}$ \\
\hline $\begin{array}{l}\text { Chile } \\
1981\end{array}$ & Trabajador & $\begin{array}{l}\text { Administrador } \\
\text { privado }\end{array}$ & $\begin{array}{l}\text { Administrador } \\
\text { privado }\end{array}$ & $\begin{array}{l}\text { Cada } \\
\text { administrador } \\
\text { tiene un fondo. } \\
\text { Esto se } \\
\text { convierte en el } \\
\text { fondo del } \\
\text { trabajador }\end{array}$ & El jubilado & $\begin{array}{l}\text { Opción de dejar } \\
\text { la cuenta con } \\
\text { la misma } \\
\text { administración o } \\
\text { de comprar una } \\
\text { anualidad de } \\
\text { una compañía } \\
\text { de seguros }\end{array}$ & $\begin{array}{l}\text { Anualidad } \\
\text { específica según } \\
\text { género o retiros } \\
\text { programados a } \\
\text { partir de la edad } \\
\text { de jubilar. Los } \\
\text { retiros pueden } \\
\text { cambiarse por } \\
\text { una anualidad }\end{array}$ \\
\hline $\begin{array}{l}\text { Australia } \\
1992\end{array}$ & Empleador & $\begin{array}{l}\text { Administrador } \\
\text { privado }\end{array}$ & $\begin{array}{l}\text { Administrador } \\
\text { privado }\end{array}$ & $\begin{array}{l}\text { Si el } \\
\text { administrador } \\
\text { elegido por el } \\
\text { empleador } \\
\text { maneja más de } \\
\text { un fondo, el } \\
\text { trabajador puede } \\
\text { elegir }\end{array}$ & El jubilado & $\begin{array}{l}\text { Opción de dejar } \\
\text { la cuenta con } \\
\text { la misma } \\
\text { administración o } \\
\text { de comprar una } \\
\text { anualidad de } \\
\text { una compañía } \\
\text { de seguros }\end{array}$ & $\begin{array}{l}\text { Suma global o } \\
\text { anualidad } \\
\text { específica por } \\
\text { género desde } \\
\text { los } 55 \text { años. } \\
\text { Beneficio de } \\
\text { sobreviviente si } \\
\text { la institución lo } \\
\text { permite }\end{array}$ \\
\hline $\begin{array}{l}\text { Reino Unido } \\
1986\end{array}$ & Trabajador & $\begin{array}{l}\text { Autoridad } \\
\text { tributaria }\end{array}$ & $\begin{array}{l}\text { Administrador } \\
\text { privado }\end{array}$ & $\begin{array}{l}\text { Los } \\
\text { administradores } \\
\text { dan a elegir } \\
\text { entre más de un } \\
\text { fondo. El obrero } \\
\text { elige }\end{array}$ & El jubilado & $\begin{array}{l}\text { Opción de dejar } \\
\text { la cuenta con } \\
\text { la misma } \\
\text { administración o } \\
\text { de comprar una } \\
\text { anualidad de } \\
\text { una compañía } \\
\text { de seguros }\end{array}$ & $\begin{array}{l}\text { Hasta } 25 \% \text { de } \\
\text { suma de pago } \\
\text { global y } \\
\text { anualidad } \\
\text { específica por } \\
\text { género desde la } \\
\text { edad de la } \\
\text { jubilación hasta } \\
\text { los } 75 \text { años. } \\
\text { Posible } \\
\text { beneficio de } \\
\text { sobreviviente }\end{array}$ \\
\hline $\begin{array}{l}\text { Suecia } \\
1995-2000\end{array}$ & Trabajador & $\begin{array}{l}\text { Autoridad } \\
\text { tributaria }\end{array}$ & $\begin{array}{l}\text { Un organismo } \\
\text { público que } \\
\text { forma parte de } \\
\text { la organización } \\
\text { de seguro social } \\
\text { y que opera } \\
\text { como cámara de } \\
\text { compensación } \\
\text { entre los } \\
\text { individuos y los } \\
\text { fondos }\end{array}$ & $\begin{array}{l}\text { El trabajador } \\
\text { elige entre } \\
\text { varios (cientos) } \\
\text { de fondos } \\
\text { mutuos inscritos }\end{array}$ & Gobierno & $\begin{array}{l}\text { Monopolio del } \\
\text { Estado / parte } \\
\text { de la } \\
\text { organización de } \\
\text { seguro social. }\end{array}$ & $\begin{array}{l}\text { Anualidad sin } \\
\text { diferencias por } \\
\text { género, parcial } \\
\text { o completa } \\
\text { desde la edad } \\
\text { de jubilación. } \\
\text { Posible } \\
\text { beneficio de } \\
\text { sobreviviente }\end{array}$ \\
\hline
\end{tabular}

Fuente: Palmer (2000).

el mercado. A fin de minimizar los costos administrativos, la administración de los activos del fondo o fondos debe asignarse por licitación. En una versión de este modelo, se permitiría a los trabajadores combinar carteras de bonos y acciones. En la versión más extrema se les obligaría a participar en una sola cartera sin posibilidad de elección individual.
El argumento a favor de la libre elección de fondos de inversión, como la que existe en Suecia, es que el Estado no debe asumir una actitud paternalista sino tan sólo garantizar, con su supervisión, que los fondos operen dentro de los límites de la buena práctica financiera. Ningún fondo podrá perder dinero siempre mientras la mayoría obtiene ganancias, y mantener a sus 
participantes. Estos estarán bien informados por la prensa y los medios de comunicación, y elegirán fondos que les ofrezcan resultados rentables. En otras palabras, el público puede tomar decisiones inteligentes. El lado opuesto de la medalla, sin embargo, es que los rendimientos de vecinos pueden ser muy distintos. Quienes sostienen la conveniencia del fondo limitado o único, creen que el público encuentra dificultad en enfrentarse al hecho de que los rendimientos de fondos distintos pueden ser desiguales. Lo que parecen olvidar, sin embargo, es que estas diferencias aparecen necesariamente entre las cohortes por edades aunque todos pertenezcan al mismo fondo, simplemente porque no todos tienen el mismo período de cotización en el fondo (Palmer, 2001).

Al final del período de inversión, se permitiría a los individuos comprar una anualidad con el capital acumulado de su pensión. Aquí también se presentan varios modelos distintos. Las anualidades podrían comprarse de un proveedor monopolista o de compañías privadas que proveen las anualidades estipuladas en la ley.

¿Cómo se han diseñado los modelos? ${ }^{15}$ En primer lugar, ¿quién elige al administrador del fondo, el trabajador o el empleador? En Chile, el Reino Unido y Suecia elige el trabajador. En Chile, cada participante tiene un fondo. En Australia y el Reino Unido elige el empleador. Éste puede contratar a un administrador de fondos que tenga a su cargo varios fondos entre los cuales pueden elegir los individuos. En el Reino Unido el administrador del fondo que el individuo contrata puede tener a su disposición más de un fondo para elegir. En Suecia, la opción se limita a cinco fondos entre los varios cientos de fondos registrados.

Otro interrogante es ¿quién elige al proveedor de las anualidades? En Chile, Australia y el Reino Unido el trabajador individual elige entre varios posibles proveedores. En Suecia, el gobierno proporciona las anualidades. Los modelos de Chile, Australia y el Reino Unido siguen más de cerca el modelo tradicional de los seguros, mientras que Suecia ha optado por privatizar los ahorros durante el período de acumulación, lo que permite a los imponentes elegir casi cualquier tipo de fondo, mientras que las anualidades son estándares para todos.

Las características de las anualidades son también distintas. En Chile, Australia y el Reino Unido se diferencian por género, mientras que en Suecia se basan

${ }^{15}$ Véase, por ejemplo, Palmer (2000) y Fox y Palmer (2001). en una esperanza de vida única para ambos géneros. El modelo sueco supone una redistribución de los hombres a las mujeres, lo que concuerda con el dictamen de la corte europea sobre la cuestión del género en las prestaciones del seguro social. Todos, menos Suecia permiten retirar una suma global. El quid está aquí en determinar si un beneficio de suma alzada se ajusta a los principios de la seguridad social, que tienen por objetivo proporcionar al jubilado la protección de un ingreso vitalicio.

Los países que han establecido sistemas de cuentas financieras individuales por las líneas que tiene Chile han conocido los altos costos de administración del sistema chileno y han intentado reducirlos con otras formas de administración. Una primera medida en ese sentido ha sido la de centralizar la recaudación de las contribuciones. Esta opción era evidente para Suecia. Las contribuciones de la seguridad social ya eran cobradas por la autoridad tributaria, de modo que el cobro de las contribuciones en esencia no supone un nuevo costo marginal.

El resto del costo marginal corresponde a la transferencia de información y dinero a los administradores de fondos. Suecia creó una caja de compensación para canalizar las contribuciones a los fondos participantes y encargarse de las transacciones netas por cuenta de los participantes con un fondo específico. En los otros tres modelos, los trabajadores individuales o sus empleadores $-\mathrm{O}$ ambos - tratan directamente con los administradores de los fondos.

Otra pregunta es quién debe mantener las cuentas y enviar los estados de cuenta. En Chile, Australia y el Reino Unido el administrador privado se encarga de esta tarea. Con el modelo sueco de caja de compensación, esta función corresponde a un organismo público (el mismo que paga las anualidades). El modelo de caja de compensación resulta eficiente para efectuar las transacciones con los administradores de los fondos, ya que el organismo puede totalizar todas las ventas y compras de un día de transacción en un solo movimiento.

En resumen, la OCDE no ha seguido a Chile en sus intentos de eliminar los sistemas de reparto y establecer sistemas de cuentas financieras. La única excepción - Australia — no tenía un sistema de reparto para comenzar, sino una amplia red de seguridad financiada por los ingresos generales del Estado, red que se esperaba reducir a medida que llegaran a la madurez los sistemas obligatorios recién establecidos de cuentas financieras administradas por los empleadores. Los países tradicionales de la OCDE tienen grandes derechos 
adquiridos bajo el sistema de reparto, similares a los de Chile en la época en que introdujo la reforma. Hasta la fecha, este hecho le ha restado interés a la posibilidad de introducir sistemas de cuentas financieras obligatorias. Donde se han introducido, las generaciones más jóvenes han transado sus derechos en el sistema de reparto por derechos en el sistema de cuentas financieras. La solución de los países europeos que han introducido sistemas de cuentas financieras ha sido la de combinarlas con el sistema de reparto, con lo cual diversifican el riesgo de los participantes entre la rentabilidad económica y la financiera.

\section{VII}

\section{La pobreza y las mujeres}

Uno de los beneficios de los sistemas aplicados en la OCDE de posguerra ha sido su impacto en la reducción de la pobreza. La mayor parte de ese impacto puede atribuirse al alto nivel de cobertura. Además, actúan otros dos factores importantes: i) las disposiciones especiales para las mujeres, y ii) redes de seguridad adicionales. La expansión de la cobertura en los sistemas de la OCDE tuvo como punto de partida la necesidad de asegurar un ingreso a las familias, y sobre todo a las viudas. El diseño de los sistemas solía incluir las siguientes prestaciones adicionales que beneficiaban casi exclusivamente a las mujeres:

- Beneficios de sobrevivencia para la cónyuge sin pensión o con una pensión más baja y para hijos menores hasta que lleguen a la mayoría de edad o se gradúen de una escuela (para compensar la pérdida del ingreso o pensión del principal sostén de la familia);

- Crédito para los efectos de la pensión por el tiempo en que se abandona el trabajo para tener a los hijos (lo que eleva la pensión de las mujeres que estarían normalmente en la fuerza trabajadora salvo durante la crianza de sus hijos); y

- Para los esquemas de cuentas financieros, anualidades sin distinción de género en regímenes de beneficios definidos o de contribuciones definidas (compensando a las mujeres por tener una esperanza de vida mayor que los hombres).

Estos factores han contribuido de manera importante a reducir la tasa de pobreza en la población de mayor edad. Muchos sistemas originalmente incluían también una edad de jubilación más temprana para las mujeres, porque las mujeres tendían a ser menores que sus esposos y se consideraba conveniente que ambos cónyuges se retiraran a un mismo tiempo. Para las mujeres, esta disposición resultó ser un arma de doble filo. En los sistemas de beneficios definidos con bajos niveles de acceso resultaban en una redistribución a favor de las mujeres. En los sistemas en que los beneficios estaban muy ligados a las cotizaciones, esta disposición podía traducirse en una menor jubilación para las mujeres por el menor tiempo de contribución.

La reducción de la pobreza también se logra con una redistribución focalizada en los sistemas para la vejez. Todos los sistemas de la OCDE incluyen algún tipo de pensión mínima. En algunos casos, se trata de un subsidio demográfico, que proporciona un ingreso mínimo a todos los que han cumplido la edad para jubilar cualesquiera sean sus contribuciones de previsión social (Suecia antes de la reforma del sistema, Dinamarca, Australia, Nueva Zelandia, Estados Unidos). En otros casos, es una pensión mínima supeditada a la exigencia de haber cumplido un número de años de cotización. El propósito del mínimo condicionado es, por un lado, aumentar el incentivo para que participen los trabajadores de menores ingresos, aumentando así la cobertura y el apoyo público para el sistema en general y, por el otro, reducir la pobreza.

$\mathrm{La}$ tendencia de la reforma europea a establecer una vinculación más fuerte entre las cotizaciones y los beneficios ha modificado, pero no ha erosionado muchos de estos beneficios. La mayoría de los sistemas han conservado el beneficio mínimo. Para las mujeres, los estatutos sobre no discriminación tienden, en la mayoría de los países, a igualar las edades de jubilación. Sin embargo, esos mismos estatutos exigen anualidades sin distinción de género, lo que significa una transferencia sobre todo a las mujeres solteras (para los matrimonios resulta en una transferencia dentro del hogar, sobre todo si existen beneficios para el sobreviviente). Muchos países acreditan en sus sistemas públicos, para efectos de la pensión, el tiempo dedica- 
do al cuidado de los hijos menores, pero no así en los sistemas vinculados a la ocupación. En Suecia el crédito por cuidado de hijos se paga con una transferencia del presupuesto general al presupuesto de la seguridad social, de modo que los fondos incluso están disponibles para la inversión en el sistema obligatorio de financiamiento por contribución definida; se espera que con la elevada participación de las mujeres en la fuerza trabajadora, así como la casi igualdad de salarios entre hombres y mujeres, junto con el crédito por cuidado del hijo para compensar a las mujeres por el tiempo que dejan de trabajar, se logrará una distribución bastante equitativa de las pensiones y una ade- cuada protección de las mujeres. En consecuencia, dicho país está eliminando gradualmente el beneficio de sobrevivencia para los cónyuges en el sistema de reparto, pero en el sistema de cuenta financiera ofrece la opción de una pensión conjunta.

Por último, la mayoría de los países de la OCDE cuentan con sistemas de asistencia social que proporcionan prestaciones en efectivo y en especie y que constituyen la red de seguridad social de última instancia. Aunque se los está eliminando, es muy posible que la tendencia a que se generalicen las pensiones ligadas a las contribuciones haga que con el tiempo estos sistemas tengan más, y no menos, importancia.

\section{VIII}

\section{Enseñanzas para América Latina}

Por razones muy similares a las de Europa, los países latinoamericanos han estado abandonando los sistemas redistributivos de beneficio definido para adoptar los basados en contribuciones. Sus metas son muy parecidas:

- reducir los gastos a futuro, elevando la edad de jubilación

- reducir el riesgo moral

- aumentar el ahorro público.

Sin embargo, han aparecido varias importantes diferencias entre las reformas latinoamericanas y las europeas. Los países europeos: i) han conservado un componente mayor de sistema de reparto, por ejemplo, que Chile y aquellos países latinoamericanos que calcaron el modelo chileno; ii) han mantenido el componente antipobreza, pero han definido más estrictamente las metas de redistribución, que son de bajo costo por efecto de la alta cobertura general del sistema; iii) han mantenido o estimulado la creación de sistemas de pensiones suplementarios de carácter ocupacional; iv) continúan incluyendo una protección especial para las mujeres, y v) buscan la manera de establecer la administración de fondos en gran escala, con lo cual lograrían economías en este aspecto crucial.

El sueño de José Piñera de cerrar el sistema de reparto público no es compartido por la mayoría de los votantes en la Europa de hoy. Por varias razones, éstos siguen valorando el sistema público de pensiones. A diferencia de los de América Latina, los de Europa han proporcionado pensiones adecuadas para la mayo- ría de los hogares, y así ha evitado privaciones en los hogares de más edad. Tanto los votantes jóvenes como los de más edad aprecian este hecho. El movimiento hacia la creación de cuentas individuales es una reforma políticamente aceptable del sistema de reparto porque en Europa, con una cobertura casi universal y una distribución más igualitaria de los salarios a lo largo de la vida laboral, el sistema de cuentas individuales proporcionará también seguridad en la vejez. El mantener un sistema de reparto parcial da mayor flexibilidad a los sistemas europeos en el caso de remezones financieros y permite que continúe alguna redistribución (hacia la mujer y los indigentes) a un costo relativamente bajo. En definitiva, resulta ampliamente aceptable un sistema que en la distribución de las pensiones sigue de cerca la de los sueldos y salarios, porque la distribución de ingresos subyacente es más pareja.

En la mayoría de los países latinoamericanos, en cambio, la distribución del ingreso es mucho más desigual. Las convulsiones macroeconómicas son de mayor envergadura y tienden a ser más frecuentes las épocas de cesantía. En consecuencia, las pensiones basadas solamente en las contribuciones pueden no ser suficientes para garantizar la seguridad en la vejez. El modelo de pensiones basado en las contribuciones, con una esperanza de vida al jubilar de unos 12 a 15 años, puede costearse sobre la base de un financiamiento total o parcialmente anticipado en la mayoría de los países, y debiera proporcionar protección adecuada a 
quienes cotizan por unos 40 años. El problema está en la población no cubierta, o cubierta en parte — aquella que tiene largos períodos sin cobertura por salidas de la fuerza laboral para criar a los hijos, por desempleo o por tiempo dedicado al sector informal entre empleos en el sector formal. La principal preocupación es cómo aumentar la cobertura y la seguridad en la vejez para abarcar a toda la actual fuerza de trabajo. La solución más probable requerirá alguna redistribución, que no puede dar el sistema de cuentas individuales por sí solo.

Los sistemas europeos ampliaron la cobertura en una época de escaso endeudamiento público, baja desigualdad de los salarios y del ingreso, y rápido crecimiento del mercado del trabajo y los salarios. Lo lograron con bajos requisitos de ingreso y pensiones mínimas. Las políticas eran redistributivas dentro de cada generación y entre generaciones, esto último por el financiamiento del sistema de reparto. A medida que se expandió la cobertura y la población envejeció, estas políticas resultaron demasiado onerosas. Como ya hemos visto, en el decenio de 1990 se atenuó la redistribución (al ser la cobertura casi universal). Las políticas sobre pensiones mínimas tienden ahora a focalizarse en mayor grado en los pobres de por vida.

Las economías latinoamericanas no tienen ahora esos factores fortuitos a su favor. Aunque la fuerza trabajadora crece con rapidez, el salario real no ha estado subiendo con celeridad en la mayoría de los países, salvo Chile, y es alta la desigualdad del ingreso. También es elevada la deuda pública, en parte por el compromiso de pagar generosas pensiones contraído por el sistema anterior. En consecuencia, el costo de una gran redistribución resultaría muy elevado si se implantara ahora con financiamiento de reparto (como ha ocurrido ya a algunos países).

Sin embargo, existe la posibilidad de aumentar la cobertura y aliviar la pobreza efectuando redistribuciones limitadas. Chile, por ejemplo, estableció una pensión mínima garantizada a los (x) años de cobertura. Bolivia estableció el Bonosol, un subsidio demográfico que se concede a los 65 años de edad. Otros países debieran considerar la posibilidad de dedicar los ingresos de la privatización a conceder una pensión mínima garantizada, quizá con la exigencia de sólo diez años de cotización, pero con una alta edad de jubilación. La clave para aplicar tal política radica en i) mantener baja la garantía y ii) mantener alta la edad de jubilación. De no ser así, se erosiona el incentivo para cotizar y el costo resulta exorbitante.

Otra forma de lograr que el sistema permanezca dentro de límites económicos razonables es desarrollar un plan complementario de pensiones vinculado a la ocupación, fuera del régimen público. No es buena idea incluir estos planes complementarios en el sistema público porque generan una transferencia de ingresos tributarios desde los hogares más pobres hacia los más ricos. En cambio, los planes organizados privadamente, incluso los organizados por los sindicatos, pueden aliviar la carga de los sistemas públicos. Pueden servir de puente con los sistemas públicos en el caso de la jubilación anticipada y pueden ser una solución para los problemas de grupos especiales, como los profesores y los empleados de la administración pública. Europa tiene dos enseñanzas que transmitir. En primer lugar, se trate de sistemas de beneficios definidos o de sistemas de contribuciones definidas, es necesaria una regulación cuidadosa. En segundo lugar, las pensiones de contribuciones definidas son menos onerosas, más fáciles de reglamentar y facilitan la movilidad de la fuerza laboral y la reestructuración económica.

Las pensiones han sido un factor importante para alejar la pobreza de la vida de muchas viudas y mujeres solteras de edad avanzada. Los sistemas de cuentas individuales, que ofrecen menos posibilidades de redistribución, podrían alterar esta situación. Adoptar la postura europea frente a los problemas de género en materia de pensiones ayudaría a los países latinoamericanos a reducir la pobreza. La mayoría de los países que han implantado sistemas de cuentas individuales establecen también una edad más temprana de jubilación para las mujeres. Como se señaló más atrás, en sistemas basados en las contribuciones esto redundará en pensiones más bajas para ellas, sobre todo cuando se combina con pensiones específicas por género. En consecuencia, serán muchas las mujeres que recibirán la pensión mínima, si es que reciben alguna. Las pensiones de sobrevivencia y las pensiones conjuntas ayudarían a contrarrestar esta tendencia. 


\section{IX}

\section{Resumen y conclusiones}

Hay importantes diferencias en la evolución económica de los países latinoamericanos y europeos y éstas han dado forma a los sistemas de pensiones y a las opciones de reforma que se abren en el umbral del siglo XXI. En Europa, la expansión de los sistemas se vinculó estrechamente con una mayor participación en los mercados del trabajo formales. La universalidad de los sistemas de pensiones y otras instituciones del mercado del trabajo se desarrollaron simultáneamente y, como resultado, disminuyó la desigualdad. Esto no ocurrió en América Latina. En consecuencia, no resultaron eficaces los intentos de lograr la cobertura universal mediante sistemas contributivos. La expansión exigía grandes trasferencias del sector público, que resultaban insostenibles. Además, los sistemas latinoamericanos se vieron afectados por algunos de los mismos problemas de jubilación anticipada que los sistemas europeos, pero financiados con una base de cotizaciones mucho menor. Los problemas financieros ocurrieron mucho más temprano y fueron más graves.

América Latina ha aprendido que los objetivos y los medios de los sistemas públicos de pensiones deben guardar coherencia con los equilibrios macroeconómicos a fin de que puedan sostenerse. Las reformas latinoamericanas han mostrado a los países europeos nuevos modos de conciliar los sistemas de pensiones con esos equilibrios fundamentales: los sistemas de cuentas financieras individuales de contribuciones de- finidas, que han dado estabilidad a los regímenes de pensiones latinoamericanos. Sin embargo, estos sistemas de hecho reproducen la desigualdad del ingreso de los trabajadores en la desigualdad de las jubilaciones y reflejan el grado de formalidad de la economía. Para reducir aún más la pobreza podrá ser necesario atender más a las causas de la pobreza en la vejez (bajos ingresos a lo largo de toda la vida, sobre todo entre las mujeres) y aplicar métodos estructurados y de bajo costo para administrar este riesgo, con financiamiento sobre una base de reparto. Entre las medidas que podrían adoptarse figuran las siguientes:

- Mayores transferencias en el sector formal de los hombres a las mujeres durante la vida laboral para que la jubilación sea adecuada. Para efectos de la jubilación, podrían incluirse créditos por crianza de los hijos o pensiones sin distinción de género;

- Jubilaciones mínimas sostenibles (muchos sistemas latinoamericanos ya las incluyen);

- Transferencias sostenibles, previa comprobación de insuficiencia de medios, a los hogares de personas mayores.

Sin embargo, todos estos mecanismos tienen que irse desarrollando lentamente y su ampliación debe ser gradual para evitar los problemas de insostenibilidad financiera con que han tropezado iniciativas anteriores.

(Traducido del inglés)
Aarts, Leo J. M.; Richard V. Burkhauser y Philip R. de Jong (1996): Curing the Dutch Disease. An International Perspective on Dutch Disability Policy Reform, Aldershot, Inglaterra, Avebury. Banco Mundial (1998): Informe sobre el desarrollo mundial, 1998, versión en CD ROM, Washington, D.C.

(1996): De la planificación centralizada a la economía de mercado. Informe del desarrollo mundial, Washington, D.C. (1995): El mundo del trabajo en una economía integrada. Informe sobre el desarrollo mundial, Washington, D.C. (1994): Averting the Old Age Crisis: Policies to Protect the Old and Promote Growth, Washington, D.C.

BID (Banco Interamericano de Desarrollo) (1998): América Latina frente a la desigualdad. Progreso económico y social en América Latina. Informe 1998-1999, Washington, D.C.

Förster, Michael (2000): Trends and Driving Factors in Income Distribution and Poverty in the OECD Area, Labour Market and Social Policy Occasional Papers, $N^{\circ}$ 42, París, Organización de Cooperación y Desarrollo Económicos (OCDE).
Fox, Louise y Edward Palmer (2001): New approaches to multipillar pension systems: what in the world is going on?, New Ideas about Old Age Security, Robert Holzmann y Joseph Stiglitz (comps.), Washington, D.C., Banco Mundial.

Gruber, Jonathan y David A. Wise (1999): Social Security and Retirement Around the World. National Bureau of Economic Research Conference Report, Chicago y Londres, University of Chicago Press.

Holzmann, Robert (1997): Pension Reform, Financial Market Development and Economic Growth: Preliminary Evidence from Chile, IMF Staff Papers, vol. 44, No 2, Washington, D.C.

Holzmann, Robert, Truman Packard y José Cuesta (2000): Extending Coverage in Multi-Pillar Pension Systems: Constraints and Hypotheses, Preliminary Evidence and Future Research Agen$d a$, Social Protection Discussion Paper, $\mathrm{N}^{\circ}$ 0002, Washington, D.C., Banco Mundial.

Ilmarinen, Juhani E. (1999): Aging Workers in the European Union, Helsinki, Instituto Finés de Salud Ocupacional. 
NSIB (National Social Insurance Board) (2001): The Dilemma of the Welfare System. Coping with Ageing Means Working Longer, Estocolmo.

oCDE (Organización de Cooperación y Desarrollo Económicos) (2000): Reforms for an Ageing Society, París.

Palmer, Edward (2001): Swedish pension reform: how did it evolve and what does it mean for the future?, Coping with the Pension Crisis: Where Does Europe Stand?, Martin Feldstein y Horst Siebert (comps.), Chicago, Illinois, University of Chicago Press.

(2000): The Swedish Pension Reform Model: Framework and Issues, Social Protection Discussion Paper, $N^{\circ}$ 0012, Washington, D.C., Banco Mundial.
(1999): Exit from the labor force of older workers: can the NDC Pension System help?, The Geneva Papers on Risk and Insurance, vol. 24, octubre.

Settergren, Ole (2001): The Automatic Balance Mechanism of the Swedish Pension System, Estocolmo, Junta Nacional de Seguridad Social.

Steuerle, Eugene, Christopher Spiro y Richard W. Johnson (1999): Can Americans work longer?, Straight Talk on Social Security and Retirement Policy, $\mathrm{N}^{\circ}$ 5, Washington, D.C., Urban Institute.

Valdés-Prieto, Salvador (1997): Financing a pension reform toward private funded pensions, The Economics of Pensions; Principles, Policies and International Experience, Cambridge, Reino Unido, Cambridge University Press. 